\title{
475743 - RECONSTRUCTIVE HEAD AND NECK SURGERY: PREDICTING COMPLICATION
}

\author{
Leonid Minkovich, $\mathrm{MD}^{1}$, Rajan Patel, $\mathrm{MD}^{2}$, Nicholas Mitsakakis, M.Sc. ${ }^{1}$, Stuart \\ McCluskey, $\mathbf{M D}^{1}$, Ralph Gilbert, $\mathrm{MD}^{2}$ \\ 1. Anesthesia, Toronto General Hospital, Toronto, ON, Canada \\ 2. Otolaryngology/Head and Neck Surgery, Toronto General Hospital, Toronto, ON, \\ Canada
}

Introduction: Wide tumor excision and free flap tissue transfer is a cornerstone in treatment of head and neck cancers. Although this surgery has high cancer cure rate, it is associated with major postoperative complications. These complications have been associated with the complexity of surgery, co-morbidities, and intra-operative management. If we can predict which patients are more likely to suffer postoperative complications then we may be able to develop targeted interventions to reduce the incidence of these complications. The objective of the study was to identify predictors of postoperative complications.

Methods: Following institutional approval, a retrospective chart review was conducted on all patients having head and neck reconstructive surgery from 1999 to 2006 at an academic institution. Perioperaive variables extracted from patients chart included: patient demographics, oncological diagnosis, comorbidity (Kaplan Fienstein Index), smoking history, medications, laboratory parameters (hemoglobin concentration $(\mathrm{Hb})$, platelet count, PTT/INR, and creatinine), and surgical variables (type of anesthesia, duration of the surgery and intraoperative fluid administered). The primary outcome variable was in hospital mortality, or major medical complication defined a prior as requiring intervention from a medical consulting service. Statistical analyses were performed using SAS version 9.1 (SAS institute, Cary, NC). Unadjusted association of potential predictors of medical complications was evaluated for both continuous (t-test) and categorical (Chi-squared test) variables. Independent predictors of outcome were identified by multivariable logistic regression analysis.

Results: The population consisted of 803 patients, 121 bone flaps and 679 tissue flaps. The incidence of medical complications was $14 \%$ (112/803) (Table). Univariate analysis identified the following predictor variables: age, preoperative hemoglobin, body mass index (bmi), volume of intraoperative fluid administered, use of tracheostomy, and history alcoholism. Independent predictors of medical complication identified by multivariate logistic regression analysis included tracheostomy (OR = 1.994; 95\% CI 1.128-3.096; $\mathrm{p}=0.002$ ), history of alcoholism (OR 1.779, 95\% CI 1.026-3.085; $\mathrm{p}=0.04$ ), age (OR 1.038, 95\% CI 1.020-1.056; $\mathrm{p}<0.001$ ), preoperative Hb (OR 0.98, 95\% CI 0.968-0.993; $\mathrm{p}=0.02$ ), and BMI (OR 958, 95\% CI 0.912-1.006; $\mathrm{p}=0.084)$. The model was well calibrated (Hosmer-Lemeshow test, $\mathrm{P}=0.610$ ) and discriminative (ROC area 0.711).

Discussion: While it is possible to predict medical complications following reconstructive surgery for head and neck cancers, in our series the only modifiable risk factor was preoperative $\mathrm{Hb}$. 
References: None

\begin{tabular}{|c|c|}
\hline Major Complication & Incidence $\mathrm{n}(\%)$ \\
\hline Mortality & $17(2.1)$ \\
\hline Cardiac & $63(7.9)$ \\
\hline Respiratory & $32(4.0)$ \\
\hline Thromboembolic & $25(3.1)$ \\
\hline Neurological & $19(2.4)$ \\
\hline Renal Failure & $3(0.4)$ \\
\hline Other & $11(1.4)$ \\
\hline
\end{tabular}

